\title{
Partición de la energía bruta consumida y el aporte de energía metabolizable en bovinos F1
}

\section{Partitioning of gross energy intake and metabolizable energy supply in bovines F1}

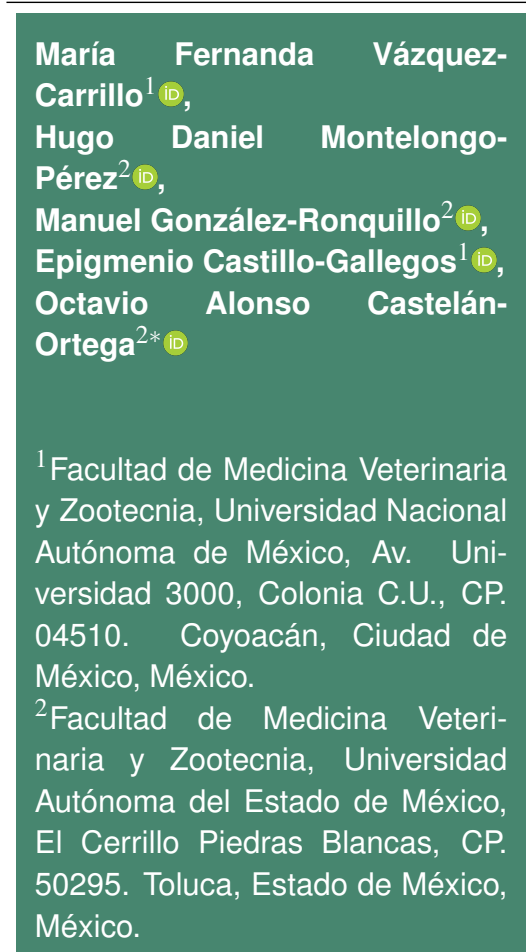

*Autor de correspondencia:

oacastelano@uaemex.mx

\section{Nota científica}

Recibida: 09 de marzo 2021 Aceptada: 04 de junio 2021

\section{Como citar: Vázquez-Carrillo}

MF, Montelongo-Pérez HD,

González-Ronquillo M, Castillo-

Gallegos E, Castelán-Ortega OA

(2021) Partición de la energía bruta consumida y el aporte de energía metabolizable en bovinos F1. Ecosistemas y Recursos Agropecuarios 8(2): e2976. DOI: $10.19136 /$ era.a8n2.2976
RESUMEN. Se estimó la partición de la energía bruta consumida (EBi) por novillonas, la densidad energética y metabolicidad de la dieta $(q m)$, y el factor de partición de la energía hacia metano $(Y m)$, por la técnica de cámaras de respiración de circuito abierto. Se utilizaron cuatro novillonas de $206 \pm 39.7 \mathrm{~kg}$ de peso vivo y $12 \pm 0.93$ meses de edad; distribuidas en un diseño completamente al azar y dos dietas: dieta $A$ con $74 \%$ de ensilado de maíz + 26\% de concentrado y dieta B con $100 \%$ de ensilado de maíz. Se utilizó una prueba de t para el análisis de resultados. El consumo de materia seca (CMS), digestibilidad, factor $Y m$ y el rendimiento de metano $\mathrm{g}$ $\mathrm{kg}^{-1}$ de CMS, para ambas dietas fue diferente $(P<0.05)$. Se logró estimar la partición de la EBi, la densidad energética y los factores $Y m$ y $q m$ de la dieta.

Palabras clave: Consumo de materia seca, digestibilidad, factor Ym, metabolicidad, metano.

ABSTRACT. The partition of the gross energy intake (GEi) by heifers, the energy density, metabolicity of the diet $(q m)$, and the $Y m$ factor were estimated, by the open circuit respiration chamber technique. Four heifers of $206 \pm 39.7 \mathrm{~kg}$ of live weight and $12 \pm 0.93$ months of age were used in a completely randomized design. The heifers were randomly assigned to two diets: Diet A consisted of $74 \%$ corn silage $+26 \%$ commercial concentrate and diet B 100\% corn silage. A t-test was used for analysis of results. Dry matter intake (DMI), digestibility of the DM, Ym factor and methane yield $\left(\mathrm{CH}_{4} \mathrm{~g} \mathrm{~kg}^{-1} \mathrm{DMI}\right)$ for both diets were different $(\mathrm{P}<0.05)$. In the present study we were able to estimate the partitioning of the GE consumed by the experimental animals; as well as the energy density, the $Y m$ and $q m$ factors of the diet.

Key words: Digestibility, dry matter intake, metabolicity, methane, Ym Factor. 


\section{INTRODUCCIÓN}

La partición y utilización de la energía de los alimentos es un factor fundamental y limitante para la productividad de los bovinos (Posada-Ochoa et al. 2017). Para medir la partición de la energía en los bovinos se utilizan técnicas calorimétricas, como ensayos metabólicos y estudios de sacrificio comparativo, las cuales permitieron el desarrollo de sistemas de alimentación (SA) para los bovinos como son el AFRC (1993), NRC (2000), CSIRO (2007), INRA (2007), entre otros. Estos SA permiten, entre otras cosas calcular las necesidades diarias de energía y predecir el balance energético del animal (Galyean et al. 2016). Asimismo, cada sistema de alimentación posee una base de datos sobre la composición química y nutricional de alimentos, especialmente sus contenidos de energía metabolizable (EM) y proteína metabolizable (PM) que permiten usar las ecuaciones del sistema para predecir la respuesta animal. Sin embargo, tales SA se desarrollaron para ambientes específicos, es decir, se crearon para condiciones climáticas particulares de los países que los desarrollaron y con insumos alimenticios locales. Por tal motivo, es cuestionable aplicar los sistemas de alimentación de otros países a nuestras condiciones geo-climáticas. Por ejemplo, las plantas que crecen en regiones de clima árido y cercanas al ecuador, en general, contienen menos material digestible (más fibra y menos proteína), que aquellas que crecen en las regiones templadas y de tundra (Lee et al. 2017, Lee 2018).

En este orden de ideas, no debe sorprender que los SA sirvan mayormente para la industria ganadera del país de origen respectivo (Sainz et al. 1994). Así, a la fecha no se cuenta con SA mundiales, debido a las diferencias y particularidades de cada región bioclimática del planeta (Sainz et al. 1994). Por ejemplo, el uso de los sistemas basados en energía neta (EN), como en el NRC (2000) utiliza pruebas de sacrificio comparativo para obtener sus valores de EN, no obstante, en la actualidad la mayoría de los valores de EN se calculan a partir de la EM utilizando ecuaciones basadas en la conversión de energía digestible (ED) a EM (Galyean et al.
2016), pero aún estos factores de partición que se consideraban como estándar están actualmente bajo revisión por lo motivos antes mencionados.

De esta forma, la EN ha sido determinada empíricamente para algunos ingredientes, aunado a que la relación entre ED y EM puede variar considerablemente entre los ingredientes del alimento o las dietas (Galyean et al. 2016). Países menos desarrollados, pero con una industria ganadera importante como Brasil, el cual cuenta con el hato comercial de bovinos más grande del mundo, ha desarrollado sus SA para ganado cebuíno y sus cruzas, tales SA se encuentran más adecuados al ganado y forrajes de las regiones de clima tropical de Brasil, ya que se reconoce que una forma de aumentar la productividad del ganado es mejorando los sistemas de cálculo de los requerimientos nutricionales (Valadares-Filho et al. 2016).

Asimismo, conociendo las transformaciones que ocurren durante el proceso de partición de la energía en el animal se puede buscar alternativas de manejo nutricional que permitan reducir las pérdidas en el sistema animal, e incrementar la cantidad de energía del alimento retenida en productos utilizables como leche o carne (Mendoza et al. 2008, Kongphitee et al. 2018). Por ejemplo, para incrementar el aporte de EM sería necesario reducir las pérdidas de energía en heces, orina y en gases como el metano $\left(\mathrm{CH}_{4}\right)$ (Mendoza et al. 2008). En este sentido, al porcentaje de energía bruta (EB) consumida convertida en metano se le conoce como factor $Y m$; la magnitud o el tamaño de este factor se encuentra influenciada por características propias de la dieta como su digestibilidad, composición química, tasa de pasaje y características propias del animal como la variación en la microbiota ruminal, consumo de materia seca (CMS) y cinética de degradación (IPCC 2006, Jaurena et al. 2015). Por lo tanto, para reducir las pérdidas de energía del alimento a partir del $\mathrm{CH}_{4}$ e incrementar la eficiencia de utilización de la energía es necesario tener en cuenta los factores antes mencionados (Hristov et al. 2013). En México existen diferentes sistemas de producción, que van desde la ganadería de traspatio, hasta sistemas con un alto grado de tecnificación; definidos por una gran diver- 
sidad de componentes, entre ellos la genética animal, la región bioclimática y por consiguiente el tipo de alimentación; ya que aproximadamente la mitad del país se encuentra en la región intertropical y la otra mitad en el hemisferio norte del planeta (Améndola et al. 2005, Castelán-Ortega et al. 2019). Por lo tanto, no existe duda que la disponibilidad de la energía de las plantas para los bovinos en función de su sitio de crecimiento (Lee et al. 2017), impacta la eficiencia de utilización de la energía de los alimentos por los bovinos, en sus requerimientos de energía, y sobre la producción de carne o leche (Améndola et al. 2005, Lee 2018). Además de la variabilidad generada por la raza, sexo y estado fisiológico del animal (NRC 2000), también la composición de los nutrientes y el valor energético de los alimentos varía ampliamente de acuerdo a las diferencias genéticas de la planta, dadas por factores como el manejo y la región climática en la que se encuentren (Lee 2018). Lo anterior, demuestra la importancia de estimar el aporte energético y partición de la energía de dietas elaboradas con ingredientes locales y bovinos de México, para desarrollar sistemas de alimentación basados en características del ganado y los forrajes y alimentos propios del país.

En México no existen sistemas de alimentación que tomen en cuenta los aspectos antes mencionados, por lo tanto, se utilizan los diferentes SA según el criterio del investigador. En este sentido el presente estudio tuvo como objetivo determinar la partición de la EB consumida por el animal, la metabolicidad de la dieta y el factor $Y m$ a través de la cuantificación de las emisiones de metano $\left(\mathrm{CH}_{4}\right)$ por la técnica de la cámara de respiración a circuito abierto, empleando ganado bovino y dos dietas características de una región de clima templado de México.

\section{MATERIALES Y MÉTODOS}

\section{Localización}

El experimento se realizó en el Laboratorio de Ganadería, Medio Ambiente y Energías Renovables (LAGMAER) de la Facultad de Medicina Veterinaria y Zootecnia de la Universidad Autónoma del Estado de México (FMVZ-UAEMex), ubicado en El Cerrillo
Piedras Blancas, Toluca, Estado de México a una latitud norte de $19^{\circ} 24^{\prime} 15^{\prime \prime}$, longitud oeste de $99^{\circ} 41^{\prime}$ 06", y a una altura de $2632 \mathrm{msnm}$.

\section{Equipo de medición}

EI LAGMAER cuenta con dos cámaras de respiración de circuito abierto, provistas de puerta delantera y trasera. Además de una válvula de entrada de aire en la parte superior; así como un ventilador para favorecer la co-dispersión de los gases, aire acondicionado y luz artificial en su interior. Los animales se mantuvieron a una temperatura y humedad relativa promedio de $18{ }^{\circ} \mathrm{C}$ y $41.7 \%$, respectivamente. Dentro de cada cámara de respiración (CR) se tiene una jaula metabólica, que tiene comedero y bebedero tipo canoa, por debajo de la jaula en la parte posterior se colocó un contenedor para colectar las heces. EI sistema de medición de $\mathrm{CH}_{4}$ estuvo compuesto por dos generadores de flujo de masas modelo FK2K, un analizador de metano por infrarrojo cercano modelo MA-10, un multiplexor (RM-8), una interface modelo UI-2 con sistema de medición a una computadora de escritorio (Vázquez-Carrillo et al. 2020, CastelánOrtega et al. 2020). Todo el sistema es de la marca Sable Systems International (Las Vegas, USA). Previo a cada medición se calibró el analizador, primero se inyectó nitrógeno de alta pureza ( $\mathrm{N}_{2}$, Praxair Inc.), hasta que el display marcó $0.000 \%$, posteriormente se inyectó una mezcla de gases con una concentración de $\mathrm{CH}_{4}$ de $0.099 \%$, una vez que el analizador de $\mathrm{CH}_{4}$ marcó la concentración conocida $\mathrm{CH}_{4}$ se consideró calibrado.

\section{Animales experimentales}

Los animales recibieron atención y manejo humanitario; el experimento fue autorizado por el Subcomité Institucional de Cuidado y Uso de Animales Experimentales, protocolo DC2018 / 2-8, de la Universidad Nacional Autónoma de México. Se utilizaron cuatro bovinos hembras F1 (Holstein $\times$ Charoláis) de $206 \pm 39.7 \mathrm{~kg}$ peso vivo (PV) promedio inicial y edad de $12 \pm 0.93$ meses. Antes de iniciar el experimento los animales se vitaminaron y desparasitaron, y se encontraron clínicamente sanos. Los animales se adaptaron a las cámaras de respiración (CR), y 
se llevaron por parejas dos veces por semana por un periodo de 8 a $10 \mathrm{~h} \mathrm{~d}^{-1}$, con la finalidad de que su CMS no se viera afectado durante el periodo de medición en las cámaras. Los animales se alojaron en corrales individuales durante todo el experimento. Al inicio y al final de cada periodo experimental los animales se pesaron previo ayuno de 14 a $16 \mathrm{~h}$.

\section{Dietas experimentales}

Se evaluaron dos tratamientos experimentales, que corresponden a dietas similares a las utilizadas en la región central del país y elaboradas con insumos locales: dieta A, compuesta por $74 \%$ de ensilado de maíz $+26 \%$ de concentrado comercial (Fatina ${ }^{\circledR}$ ), y la dieta B compuesta por $100 \%$ de ensilado de maíz. La composición química del ensilado de maíz fue $33 \% \mathrm{MS}, 76.8 \mathrm{~g}$ de proteína cruda $\mathrm{kg}^{-1}$ MS (PC), $556.8 \mathrm{~g}$ de fibra detergente neutro $\mathrm{kg}^{-1} \mathrm{MS}$ (FDN) y $255.5 \mathrm{~g}$ de fibra en detergente ácido $\mathrm{kg}^{-1}$ MS (FDA). La composición química del alimento concentrado fue de $88 \% \mathrm{MS}, 120 \mathrm{~g} \mathrm{PC} \mathrm{kg}^{-1} \mathrm{MS}, 331 \mathrm{~g}$ FDN kg ${ }^{-1} \mathrm{MS}$, y $114.2 \mathrm{~g} \mathrm{FDA} \mathrm{kg}^{-1} \mathrm{MS}$.

\section{Mediciones}

Los animales fueron alimentados una vez al día a las 9:00 AM, ofreciendo 8 y $7 \mathrm{~kg}$ MS de la dieta $\mathrm{A}$ y $\mathrm{B}$, respectivamente, y el agua se ofreció ad libitum. Todos los días se pesó la dieta ofrecida y se tomó una muestra del $5 \%$ del alimento ofrecido a cada animal para tener alícuotas representativas. El CMS se estimó por diferencia del alimento ofrecido menos el rechazado. Para el cálculo de la digestibilidad aparente de la dieta (DIG), se midió la producción total de heces de cada animal; las cuales se recolectaron con una pala directamente de la jaula metabólica que estaba dentro de la CR, todas las heces se pesaron y se tomó una muestra de aproximadamente el $10 \%$ del total de heces producidas por animal. Las alícuotas de dietas y heces, se secaron en una estufa de aire forzado (Ecoshel 9023A, México) a $60{ }^{\circ} \mathrm{C}$ por 72 y $120 \mathrm{~h}$, respectivamente (AOAC 1990). Una vez determinada la MS se preservaron en bolsas individuales dentro de un recipiente, ambos de plástico, para su posterior análisis de energía bruta (EB, MJ/kg MS) por medio de una bomba calorimétrica de marca Parr (Illonois, USA), la PC se determinó por el método de Kjeldahl (AOAC 1990) y el contenido de fibra detergente neutro (FDN) y ácida (FDA) con el analizador de fibras ANKOM 200 (ANKOM, USA).

\section{Determinación de la partición de la energía bruta consumida}

Se obtuvo el valor calorífico del alimento y las pérdidas de energía en forma de heces y $\mathrm{CH}_{4}$. La producción total de $\mathrm{CH}_{4}$ por animal $\left(\mathrm{ECH}_{4}\right)$ se multiplicó por su valor calorífico, asumiendo que $1 \mathrm{~g}$ de $\mathrm{CH}_{4}$ equivale a $55.5 \mathrm{~kJ}$ (Brouwer 1965). No se recolectó la orina, por lo cual su contenido energético se estimó suponiendo que del total de la EB ingerida, el 3 a 5\% (Van Soest 1994) se pierde en la orina, se tomó como referencia el 3 y $4 \%$ para dietas con baja y alta digestibilidad, respetivamente (Kirkpatrick et al.1997). La energía digestible consumida (EDi, $\mathrm{MJ} \mathrm{d}^{-1}$ ) se determinó por la diferencia entre la EB ingerida (EBi) menos la EB contenida en las heces. La $\mathrm{EM}$ ingerida (EMi, $\mathrm{MJ} \mathrm{d}^{-1}$ ) fue el resultado de la EDi menos la energía contenida en la orina y el $\mathrm{CH}_{4}$; y la metabolicidad de la dieta el resultado de dividir la EM entre la EB (AFRC 1993). La densidad energética de la dieta en términos de ED y EM, expresada en MJ $\mathrm{kg} \mathrm{MS}^{-1}$, se obtuvo al multiplicar la EB de la dieta por el porcentaje de la EDi y EMi divididos entre EBi, respectivamente. Finalmente, el factor $Y m(\%)$ se obtuvo dividiendo $\mathrm{ECH}_{4}$ entre la $\mathrm{EBi}$.

\section{Diseño experimental y análisis estadístico}

Los cuatro animales fueron distribuidos en un diseño completamente al azar, dos animales en cada tratamiento experimental, en cada animal se midieron las variables de estudio dos veces (8 submuestras) al final de cada periodo de medición. En cada ocasión los animales tuvieron 24 días de adaptación a la dieta y cuatro días de medición. De esta forma dos animales se alimentaron con la dieta $A$, y dos animales con la dieta B. En los días de medición los animales se llevaron a las CR para cuantificar la emisión de $\mathrm{CH}_{4}$ durante $24 \mathrm{~h}$ (un animal por cámara), el CMS y la DIG. De esta forma se obtuvieron dos mediciones de cada variable por animal. Los resultados 
se analizaron con el siguiente modelo mixto y lineal según Kuehl (1999):

$$
Y_{i j k}=\mu+\tau_{i}+\varepsilon_{i j}+d_{i j k}
$$

Donde $\mu$ es la media general, $\tau_{i}$ es el efecto fijo del i-ésimo tratamiento, $\varepsilon_{i j}$ es el efecto aleatorio del error experimental para la j-ésima unidad experimental del i-ésimo tratamiento, y $d_{i j k}$ es el efecto aleatorio para la k-ésima submuestra de la j-ésima unidad experimental del i-ésimo tratamiento (distribución normal, $\mu=0, \sigma=1)$. Para el análisis de varianza se utilizó el software RStudio versión 1.3.1073 (R core Team 2019).

\section{RESULTADOS Y DISCUSIÓN}

La Tabla 1 muestra los resultados para las variables relativas a la partición de la energía y la emisión de $\mathrm{CH}_{4}$. Es importante indicar que el uso de un tamaño de muestra pequeño puede llegar a generar intervalos de confianza amplios para los estimadores de las medias poblacionales. Lo que consecuentemente dificulta encontrar diferencias estadísticamente significativas en las comparaciones de medias de los tratamientos (Kuehl 1999), lo que no ocurrió en el presente estudio. Se observó que el CMS de la dieta $A$ fue mayor $(P<0.05)$ con respecto a la $B$, lo cual se podría explicar porque el $26 \%$ de concentrado en la dieta $A$ favoreció una mayor digestibilidad $(P<0.05)$ y por lo tanto un mayor aporte de energía de la dieta (Blaxter 1964). También es posible que la suplementación energética del concentrado favoreciera la actividad microbiana ruminal, incrementando la digestibilidad, la velocidad de pasaje y por ende el CMS (Carvalho et al. 2020). Las propiedades físicas y químicas del forraje en la dieta $\mathrm{B}$, como son un mayor contenido de FDN, FDA, lignina, menor digestibilidad (DIG $=57.7 \%$ ), y por lo tanto menor disponibilidad de EM, posiblemente deprimieron el consumo voluntario por la resistencia del forraje a ser degradado por la masticación durante la comida y rumia, menor actividad microbiana, así como por el volumen limitado del retículorumen, de lo cual depende la velocidad de paso (Carvalho et al. 2020). Con respecto a la producción de
$\mathrm{CH}_{4}$, se observa que ambas dietas tuvieron el mismo volumen de emisión diario de $\mathrm{CH}_{4}(\mathrm{P}>0.05)$; pero el rendimiento de $\mathrm{CH}_{4}$ (g de $\mathrm{CH}_{4} \mathrm{~kg}^{-1} \mathrm{CMS}$ ) fue menor $(\mathrm{P}<0.05)$ en la dieta $\mathrm{A}, 15.4 \mathrm{~g}$ de $\mathrm{CH}_{4} \mathrm{~kg}^{-1} \mathrm{CMS}$, que el de la dieta $\mathrm{B}, 20.7 \mathrm{~g}$ de $\mathrm{CH}_{4} \mathrm{~kg}^{-1} \mathrm{CMS}$, a pesar de que el CMS fue mayor en la dieta $A(P<0.05)$. La Tabla 1 muestra también que los animales con la dieta $\mathrm{B}$, perdieron más energía en forma de $\mathrm{CH}_{4}$ con un $Y m=7.4 \%(P<0.05)$, respecto a la dieta $A, Y m=$ $5.7 \%$.

Tabla 1. Consumo, digestibilidad, producción de metano y partición de la energía bruta consumida en bovinos F1 alimentados con dos dietas.

\begin{tabular}{|c|c|c|c|}
\hline Variable $n=4$ & Dieta A & Dieta B & Valor $\mathrm{P}$ \\
\hline $\mathrm{CMS} \mathrm{kg} \mathrm{d}^{-1}$ & $6.4^{a} \pm 0.4$ & $4.6^{b} \pm 0.4$ & 0.024 \\
\hline DIG, \% & $74.7^{a} \pm 0.05$ & $57.7^{b} \pm 0.03$ & 0.050 \\
\hline CMS DIG kg d ${ }^{-1}$ & $4.7^{a} \pm 0.6$ & $2.5^{b} \pm 0.3$ & 0.037 \\
\hline $\mathrm{CH}_{4}, \mathrm{~L} \mathrm{~d}^{-1}$ & $137.7 \pm 4.8$ & $133 \pm 18.3$ & 0.415 \\
\hline $\mathrm{CH}_{4}, \mathrm{~L} \mathrm{~kg}^{-1} \mathrm{CMS}$ & $21.5^{b} \pm 1.6$ & $28.9^{a} \pm 4.5$ & 0.032 \\
\hline $\mathrm{CH}_{4}, \mathrm{~g} \mathrm{~kg}^{-1} \mathrm{CMS}$ & $15.4^{b} \pm 1.2$ & $20.7^{a} \pm 3.2$ & 0.031 \\
\hline$Y m, \%$ & $5.7^{b} \pm 0.6$ & $7.4^{a} \pm 1.2$ & 0.034 \\
\hline $\mathrm{EBi}, \mathrm{MJ} \mathrm{d}^{-1}$ & $95.7^{a} \pm 9.6$ & $71.7^{b} \pm 5.3$ & 0.024 \\
\hline $\mathrm{Eh}, \mathrm{MJ} \mathrm{d}^{-1}$ & $23.7 \pm 4.0$ & $29.3 \pm 2.2$ & 0.231 \\
\hline EDi MJ d $d^{-1}$ & $72.0^{a} \pm 9.9$ & $42.3^{b} \pm 5.3$ & 0.036 \\
\hline ED:EB & $0.75^{a} \pm 0.05$ & $0.59^{b} \pm 0.04$ & 0.050 \\
\hline Eu MJ d $d^{-1}$ & $3.83^{a} \pm 0.4$ & $2.15^{b} \pm 0.2$ & 0.006 \\
\hline $\mathrm{ECH}_{4} \mathrm{MJ} \mathrm{d}^{-1}$ & $5.45 \pm 0.2$ & $5.3 \pm 0.7$ & 0.461 \\
\hline $\mathrm{ECH}_{4}: \mathrm{EB}(Y m)$ & $0.06^{b} \pm 0.00$ & $0.07^{a} \pm 0.01$ & 0.041 \\
\hline EMi, MJ d $d^{-1}$ & $62.7^{a} \pm 9.7$ & $34.9^{b} \pm 5.3$ & 0.041 \\
\hline EM:EB & $0.65 \pm 0.05$ & $0.49 \pm 0.05$ & 0.061 \\
\hline EM:ED & $0.87^{a} \pm 0.02$ & $0.83^{b} \pm 0.03$ & 0.046 \\
\hline \multicolumn{4}{|c|}{$\begin{array}{l}\text { Dieta } \mathrm{A}=74 \% \text { de ensilado de maíz }+26 \% \text { de concentrado; Dieta } \\
\mathrm{B}=100 \% \text { de ensilado de maíz; CMS = Consumo de materia } \\
\text { seca; } \mathrm{DIG}=\text { Digestibilidad, } Y m=\text { factor de partición de la energía } \\
\text { bruta consumida hacia metano. } \mathrm{EBi}=\text { energía bruta ingerida, Eh } \\
\text { = energía perdida en la materia fecal, EDi = energía digestible in- } \\
\text { gerida, EDi:EBi }=\mathrm{Relación}_{\mathrm{E}} \mathrm{E} / \mathrm{EBi} ; \mathrm{Eu}=\text { energía perdida en la } \\
\text { orina (calculada), } \mathrm{ECH}_{4}=\mathrm{Energía} \mathrm{perdida} \mathrm{como} \mathrm{metano;} \mathrm{EMi} \\
=\text { energía metabolizable ingerida, EMi:EBi = relación EMi/EBi, } \\
\mathrm{EMi}: \mathrm{EDi}=\text { relación EMi/EDi; } Y m=\text { factor de partición de la energía } \\
\text { bruta consumida hacia metano. Todas las variables anteriores } \\
\text { fueron resultado de las mediciones dentro de las cámaras de res- } \\
\text { piración. }\end{array}$} \\
\hline
\end{tabular}

Por lo tanto, el rendimiento de $\mathrm{CH}_{4}$ dependió de la calidad de la dieta expresada en términos de su digestibilidad, está claramente establecido que las raciones basadas únicamente en forrajes presentan una fermentación mayormente de tipo acética, a diferencia de una dieta compuesta a base de grano y forraje, la cual produce mayor cantidad de ácido propiónico (Popova et al. 2011). También se sabe que la producción de ácido acético promueve la pro- 
ducción de $\mathrm{CH}_{4}$ debido a que libera moléculas de carbono en forma de $\mathrm{CO}_{2}$ y de $\mathrm{H}_{2}$, mientras que la producción de ácido propiónico conserva las moléculas de carbono y captura hidrógeno, reduciendo la emisión de dicho gas (Hook et al. 2010). Al parecer, este último mecanismo tuvo un efecto más importante en el presente experimento (Tabla 1), aun cuando la cantidad de concentrado utilizado fue de sólo $26 \%$. Asimismo, el factor $Y m$ obtenido para ambas dietas se encuentra dentro de valores encontrados para ganado de carne alimentado con dietas altas en forraje ( $>25 \%$ de inclusión de forraje) el cual oscila de $6.3 \pm 1.4$ (Van Lingen et al. 2019).

Con respecto a la partición de la energía bruta ingerida (EBi), se observó que la inclusión de cantidades pequeñas de concentrado en la dieta $A$ resultó en un consumo más elevado de $E B(P<0.05)$, a pesar de que la concentración de EB por kilogramo de MS fue igual entre ambas dietas $(P>0.05)$ (Tabla 2); sin embargo la mayor EBi se debió al CMS más alto en los animales con la dieta A (Tabla 1). De igual forma, la disponibilidad de la energía metabolizable fue mayor en la dieta $A$, ya que el factor de partición de la energía digestible a energía metabolizable (EM:ED) fue mayor en la dieta $A$ con respecto a la dieta $B(P<0.05)$, lo cual se debió al mayor consumo, digestibilidad y consumo de EB más alta de la dieta $A$ con respecto a la dieta $B$.

Tabla 2. Densidad energética y metabolicidad de las dietas experimentales.

\begin{tabular}{lccc}
\hline Variable & Dieta A & Dieta B & Valor $\mathrm{P}$ \\
\hline $\mathrm{EB}, \mathrm{MJ} \mathrm{kg} \mathrm{MS}^{-1}$ & $14.9 \pm 0.9$ & $15.5 \pm 0.2$ & 0.590 \\
$\mathrm{ED}, \mathrm{MJ} \mathrm{kg} \mathrm{MS}^{-1}$ & $11.2 \pm 1.1$ & $9.2 \pm 0.6$ & 0.073 \\
$\mathrm{EM}, \mathrm{MJ} \mathrm{kg} \mathrm{MS}^{-1}$ & $9.7 \pm 1.1$ & $7.5 \pm 0.8$ & 0.071 \\
$\mathrm{qm}$ & $0.65 \pm 0.05$ & $0.49 \pm 0.05$ & 0.060 \\
\hline Dieta $\mathrm{A}=74 \%$ de ensilado de maíz $+26 \%$ de concentrado, \\
Dieta $\mathrm{B}=100 \%$ de ensilado de maíz, EB = Energía bruta, \\
$\mathrm{ED}=$ Energía digestible, EM = Energía metabolizable; $q m=$ \\
metabolicidad de la dieta.
\end{tabular}

Todos los factores que afecten la digestibilidad, como el nivel de fibra en la dieta, van a afectar el consumo de energía digestible; por ejemplo, se ha demostrado que altos niveles de FDN en la dieta afectan de forma negativa el consumo de ED (Tarazona et al. 2012, Carvalho et al. 2020). En este sentido, la relación de EM:ED, EM:EB y ED:EB aumentará cuanto mayor sea la digestibilidad de la dieta y menor sea su concentración de FDN y FDA, y por el contario, con altos niveles de FDN y FDA, la digestibilidad de la dieta es menor y por lo tanto su aporte de EM al animal también (AFRC 1993).

La media de la relación EM:ED es de 0.87 para la dieta $A$ y 0.83 para la dieta $B$, aunque se encuentra cercano a lo sugerido por distintos sistemas de alimentación: 0.81 (CSIRO 2007), 0.82 (NRC 2000) y 0.81-0.86 (AFRC 1993), era de esperarse que fuese diferente, ya que éste valor se encuentra definido, principalmente, por el tipo de dieta, que en el caso de México tiende a ser de menor calidad sobre todo en el caso de los forrajes por el efecto del clima más cálido sobre el crecimiento y maduración de las plantas (Castelán-Ortega et al. 2019). En este sentido, nuestro valor de EM:ED es más parecido al reportado por Da Fonseca et al. (2019) para las regiones de clima tropical de Brasil, el cual fue de 0.86 para una dieta compuesta de 50:50 concentrado:forraje, sorgo y Panicum máximum cv. Tanzania.

La Tabla 2 muestra la densidad energética y metabolicidad de la dieta ( $q m)$. La ED, EM y el qm fue numéricamente diferente, siendo mayor en la dieta $A$, respecto a la dieta $B$. Este resultado se puede explicar por la mayor digestibilidad de la dieta $A(P<$ 0.05 ), ya que la digestibilidad es la principal determinante de la metabolicidad de la dieta, pues está claramente establecido que raciones más digestibles son utilizadas por los animales de modo más eficiente que las dietas de baja digestibilidad (Blaxter 1964). Los resultados para el valor $q m$ son muy parecidos a los valores más bajos de qm recomendados por el AFRC (1993) para ganado productor de carne alimentados con dietas altas en fibras $(q m=0.4)$ como la dieta $B$ y dietas de mejor calidad $(q m=0.5)$ como la dieta $A$. Esto quiere decir que la metabolicidad de los forrajes y dietas empleadas en el presente estudio son más parecidas a los forrajes de mala calidad en un país de clima templado (Lee 2018).

El presente estudio permitió determinar la partición de la $\mathrm{EBi}$, la metabolicidad y el factor $Y$ m, de los bovinos bajo estudio. Los resultados derivados de la partición de la energía, como la relación EM:ED o 
factor de partición de la energía digestible a energía metabolizable de la dieta, que, aunque se encontró cercano a lo establecido por sistemas energéticos internacionales, se encuentra por arriba del valor por defecto de 0.82 mencionado por el NRC (2000). Lo que sugiere la necesidad de generar valores acordes a las condiciones de la ganadería de cada país, tal como lo hace Brasil (da Fonseca et al. 2019), y Hales et al. (2014) quienes reportan un valor de 0.89 a 0.92 , el cual atribuyen a la dieta, a la genética, a la edad del animal, entre otros factores. Incluso Galyean et al. (2016) en una revisión hecha al Sistema de Energía Neta de California aconseja dejar de utilizar el valor por defecto de 0.82 que sugiere NRC (2000), y moverse al uso de una ecuación lineal más precisa para calcular el factor de partición EM:ED. Aunque no se observó diferencia en la densidad de la energía bruta de las dietas, la inclusión de una cantidad pequeña de concentrado mejoró la digestibilidad de la MS en la dieta $A$, haciendo más eficiente el uso de la energía por el animal. Lo que se reflejó en un valor menor para el factor $Y m$, por lo tanto es necesario realizar más estudios de este tipo utilizando diferentes forrajes y suplementos energéticos que permitan encontrar combinaciones de ambos ingredientes que hagan más eficiente el uso de la energía por los bovinos y de esta forma tener sistemas de producción más sustentables y amigables al medio ambiente.

\section{AGRADECIMIENTOS}

Al Consejo Nacional de Ciencia y Tecnología (CONACyT) por financiar parte de este trabajo a través del proyecto con clave CB2013-223418-T, así como también por la beca para estudios de doctorado de la primera autora.

\section{LITERATURA CITADA}

AFRC (1993) Energy and Protein Requirements of Ruminants. An Advisory Manual Prepared by the Agricultural Food and Research Council Technical Committee on Responses to Nutrients. CAB International. Wallingford, UK. 176p.

Améndola R, Castillo GE, Martínez PA (2005) Country pasture/forage resource profiles. México. Country pasture profiles. FAO. Roma, Italia. 68p.

AOAC (1990) Official Methods of Analysis. 15th Edition. Association of Official Analytical Chemists. Arlington, VA, USA. 1230p.

Blaxter KL (1964) Energy Metabolism of Ruminants. Hutchinson and Co. (Publishers) Ltd London, England.

Brouwer E (1965) Report of sub-committee on constants and factors. In: Blaxter KL (ed) Energy metabolism of farm animals. Academic Press. London. pp: 441-443

Carvalho HV, Pinto CJ, Millen DD, Felix TL (2020) Effect of cattle breed and basal diet on digestibility, rumen bacterial communities, and eating and rumination activity. Journal of Animal Science 98: skaa114. DOI: 10.1093/jas/skaa114

Castelán-Ortega OA, Ku-Vera JC, Ángeles-Hernández JC, Benaouda M, Hernández-Pineda GS, Molina TL, Ramírez-Cancino L, Castelán-Jaime SV, Praga-Ayala AR, Lazos-Balbuena F, Montelongo-Pérez HD, González-Ronquillo M, Vázquez-Carrillo MF, Cardoso-Gutiérrez E, Aranda-Aguirre E, Villegas-Estrada D, Guadarrama-López P, Apodaca-Martínez G (2019) Ganadería. En: Paz-Pellat F, Hernández-Ayón JM, SosaÁvalos R, Velázquez-Rodríguez AS (ed). Estado del ciclo del carbono en México: Agenda azul y verde. Programa Mexicano del Carbono. Texcoco, Estado de México, México. pp: 492-528.

Castelán-Ortega OA, Pedraza-Beltrán PE, Hernández-Pineda GS, Benaouda M, González-Ronquillo M, T Molina L, Ku-Vera JC, Montelongo-Pérez HG, Vázquez-Carrillo MF (2020) Construction and operation of a respi- 
ration chamber of the head-box type for methane measurement from cattle. Animals 10(2): 227. DOI: 10.3390/ani10020227.

CSIRO (2007) Nutrient requirements of domesticated ruminants. CSIRO Publishing. Collingwood, Australia. 270p.

da Fonseca MP, Borges ALDCC, Carvalho PHA, Silva RR, Gonçãlves LC, Borges I, Lage FH, Ferreira LA, Saliba SEO, Jayme GD, da Glória RJ, Graça SD, Meneses MR, Carvalho UA, Filho FJE, Silva AA (2019). Energy partitioning in cattle fed diets based on tropical forage with the inclusion of antibiotic additives. PLoS One. 14(4): e0211565. DOI: 10.1371/journal.pone.0211565.

Galyean ML, Cole NA, Tedeschi LO, Branine ME (2016) Board-invited review: Efficiency of converting digestible energy to metabolizable energy and reevaluation of the California Net Energy System maintenance requirements and equations for predicting dietary net energy values for beef cattle. Journal Animal Science 94: 1329-1341.

Hales KE, Brown-Brandl TM, Freetly HC (2014). Effects of decreased dietary roughage concentration on energy metabolism and nutrient balance in finishing beef cattle. Journal Animal Science 92: 264-71. DOI: 10.2527/ jas.2013-6994.

Hook SE, Denis A, Wright G, McBride BW (2010) Methanogens: Methane producers of the rumen and mitigation strategies. Archaea 2010: 945785. DOI: 10.1155/2010/945785

Hristov AN, Oh J, Lee C, Meinen R, Montes F, Ott T, Firkins J, Rotz A, Dell C, Adesogan A, Yang W, Tricarico J, Kebreab E, Waghorn G, Dijkstra J, Oosting S (2013) Mitigation of greenhouse gas emissions in livestock production - A review of technical options for non-CO2 emissions. FAO Animal Production and Health Rome, Italy. 149p.

INRA (2007) Institut National de la Recherche Agronomique France. Alimentation des bovins, ovins et caprins. Besoins des animaux. Valeur des aliments. Tables INRA 2007. Editions Quae, Versailles, France. 330p.

IPCC (2006) Guidelines for national greenhouse gas inventories. Prepared by the National Greenhouse Gas Inventories. Japan. Programme Intergovernmental Panel on Climate Change. IGES, Japan. pp. 10.110.34 .

Jaurena G, Cantet JM, Arroquy IJ, Palladino RA, Wawrzkiewicz M, Colombatto D (2015) Prediction of the Ym factor for livestock from on-farm accessible data. Livestock Science 177: 52-62.

Kirkpatrick DE, Steen R, Unsworth EF (1997) The effect of differing forage: concentrate ratio and restricting feed intake on the energy and nitrogen utilization by beef cattle. Livestock Production Science 51: 151-164.

Kongphitee K, Sommart K, Phonbumrung T, Gunha T, Suzuki T (2018) Feed intake, digestibility and energy partitioning in beef cattle fed diets with cassava pulp instead of rice straw. Asian-Australasian Journal of Animal Sciences 31: 1431-1441.

Kuehl RO (1999) Design of Experiments: Statistical Principles of Research Design and Analysis. 2nd Edition. Duxbury Press, USA. 666p.

Lee MA, Davis AP, Chagunda MGG, Manning P (2017) Forage quality declines with rising temperatures, with implications for livestock production and methane emissions. Biogeosciences 14: 1403-1417.

Lee MA (2018) A global comparison of the nutritive values of forage plants grown in contrasting environments. Journal of Plant Research 131: 641-654.

Mendoza G, Plata F, Espinosa R, Lara A (2008) Manejo nutricional para mejorar la eficiencia de utilización de la energía en bovinos. Universidad y Ciencia 24: 75-87. 
NRC (2000) Nutrient Requirements of Beef Cattle. 6th Edition. National Academy Press, Washington DC, USA. $248 p$.

Popova M, Morgavi D, Doreau M, Martin C (2011) Production de méthane et interactions microbiennes dans le rumen. INRA Productions Animales 24: 447-460.

Posada-Ochoa SL, Noguera RR, Rodríguez NM, Costa AL, Reis R (2017) Indirect calorimetry to estimate energy requirements for growing and finishing Nellore bulls. Journal of Integrative Agriculture 16: 151-161.

R core Team (2019) R: A Language and Enviroment for Statisctical Computing (version 1.3.1073). R Foundation for Statistical Computing: Vienna, Austria. https://www.R-project.org/. Fecha de consulta 5 de enero de 2021.

Sainz RD, Fernández C, Baldwin RL (1994) Valoración de alimentos para rumiantes en cebo: el sistema americano NRC. En: Rebollar PG, Mateos GG, De Blas C (eds.) X Curso de especialización FEDNA: Avances en Nutrición y Alimentación Animal. Madrid, España. pp: 227-239.

Tarazona AM, Ceballos MC, Naranjo JF, Cuartas CA (2012) Factores que afectan el comportamiento de consumo y selectividad de forrajes en rumiantes. Revista Colombiana de Ciencias Pecuarias 25: 473-487.

Valadares-Filho SC, Costa e Silva LF, Pies-Gionbelli M, Pizzi-Rotta P, Marcondes MI, Chizzotti ML, Franco-Prados L (2016) BR - Corte: nutrient requirements of Zebu and crossbred cattle. 3rd Edition. Universidade Federal de Viçosa, Viçosa Brasil. 314p.

Van Lingen HJ, Niu M, Kebreab E, Valadares FSC, Rooke J, Duthied CA, Schwarm A, Kreuzer M, Hynd IP, Caetano M, Eugéne M, Martin C, McGee M, O’Kiely P, Hünerberg M, McAllister TA, Berchielli TT, Messana DJ, Peiren N, Chaves Va, Charmley E, Cole AN, Hales EK, Lee SS, Berndt A, Reynolds CK, Crompton AL, Bayat RA, Yáñez-Ruiz DR, Yu Z, Bannik A, Dijkstra J, Casper PD, Hristov NA (2019) Prediction of enteric methane production, yield and intensity of beef cattle using an intercontinental database. Agriculture, Ecosystems \& Environment 283: 106575. DOI: 10.1016/j.agee.2019.106575

Van Soest PJ (1994) Nutritional Ecology of the Ruminant. 2nd Edition. Cornell University Press New York, USA. $476 p$.

Vázquez-Carrillo MF, Montelongo-Pérez HD, González-Ronquillo M, Castillo-Gallegos E, Castelán-Ortega OA (2020) Effects of three herbs on methane emissions from beef cattle. Animals 10: 1671. DOI: 10.3390/ani 10091671 